[7] Zur Nomenklatur siehe: G. R. Newkome, G. R. Baker, J. K. Young, J. G. Traynharn, J. Polym. Sci. Part A: Polym. Chem. 1993, 31, 641; [1 b], S. $169-$ 186.

[8] C. J. Hawker, J. M. J. Fréchet, J. Am. Chem. Soc. 1990, 112, 7638; K. L. Wooley, C. J. Hawker, J. M. J. Fréchet, J. Chem. Soc. Perkin Trans. 1 1991, 1059

[9] J. Zimmermann, D. Seebach, T.-K. Ha, Helv. Chim. Acta 1988, 7t, 1143.

[10] W. Amberg, D. Seebach, Chem. Ber. 1990, 123, 2413.

[11] Bedingungen: 1 Äquiv. Triol, 9 Äquiv. NaH in THF, 3.5 Äquiv. Astbromid, $0^{\circ} \mathrm{C}$ bis Rückfluß-Temperatur.

[12] Es wurde nicht bewiesen, daß in $\mathbf{1 3 b}$ tatsächlich die OH-Gruppe mit benachbarter $t \mathrm{Bu}$-Gruppe unverethert vorliegt.

[13] Wie schon mit anderen größeren Dendrimeren beobachtet, zeigt 11 ein ungewöhnliches Laufverhalten bei der Chromatographie; vgl. Z. Xu, M. Kahr, K. L. Walker, C. L. Wilkins, J. S. Moore, J. Am. Chem. Soc. 1994, 116, 4537.

[14] K. Nakanishi, N. Berova, R, W. Woody, Circular Dichroism, VCH, New York, 1994.

\section{Ein selbstassoziierender difunktioneller Rezeptor}

Dmitry M. Rudkevich*, Alexander N. Shivanyuk, Zbigniew Brzozka, Willem Verboom und

David N. Reinhoudt*

Die molekulare Selbstorganisation ist ein wichtiger Faktor in Lebensprozessen und biologischen Systemen ${ }^{[1]}$. Sie führt zu komplexen Strukturen, wie doppelsträngiger DNA, viralen Proteinhüllen, Lipidmembranen und globulären Proteinen. Das Konzept der Selbstorganisation wurde auch beim Design von Nanostrukturen, wie anorganischen Clustern, Röhren und Kanälen, Monoschichten sowie über $\mathrm{H}$-Brücken gebundenen Netzwerken angewendet. Die Funktionen solcher Assoziate können aus den Moleküleigenschaften abgeleitet werden ${ }^{[2,3]}$. In jüngster Zeit berichteten Sessler et al. über den photoinduzierten Energietransfer in nichtkovalent gebundenen Photosystemen $^{[4]}$. Auch konnte eine molekulare Erkennung durch die Einlagerung von kleinen Gastmolekülen in Hohlräume eines Wirtassoziates erzielt werden ${ }^{[5]}$, das durch nichtkovalente Wechselwirkungen gebildet wird. Durch Selbstorganisation aus cyclischen Peptiduntereinheiten gebildete Nanoröhren können Glucose durch Lipiddoppelschichten transportieren ${ }^{[6]}$.

Wir haben difunktionelle Rezeptoren beschrieben, die in demselben Molekül über kovalent aneinander gebundene Kationen- und Anionenbindungsstellen verfügen ${ }^{[7]}$. Hier beschreiben wir die molekulare Erkennung von Ionen durch einen difunktionellen Rezeptor, in dem eine Kationen- und eine Anionenbindungsstelle durch Selbstorganisation in unmittelbare Nachbarschaft gebracht wurden. Ein solcher Rezeptor kann gleichzeitig kationische und anionische Gastspezies binden. Die verwendete Strategie beruht auf der Assoziation von bekannten monofunktionellen Rezeptoren über Wasserstoffbrückenbindungen. Der Kationenrezeptor Calix[4]aren, der eine Amidund drei Estergruppen enthält, komplexiert Alkalimetallkationen und weist eine hohe Selektivität für $\mathrm{Na}^{+}$auf ${ }^{[8]}$. Dieses Ca-

[*] Dr. D. M. Rudkevich, Prof. Dr. Ir. D. N. Reinhoudt, Dr. A. N. Shivanyuk, Dr. habil. Z. Brzozka ${ }^{l+}$, Dr. W. Verboom

Laboratory of Organic Chemistry, University of Twente

P.O. Box 217. NL-7500 AE Enschede (Niederlande)

Telefax: Int. $+53 / 312738$

$\left[^{+}\right]$Ständige Adresse:

Department of Analytical Chemistry, Technical University of Warsaw (Polen) lixaren wurde aus dem entsprechenden Triester-Monocarbonsäurechlorid ${ }^{[9]}$ und 2-Amino-6-(hexanamido)pyridin in 78\% Ausbeute hergestellt. Der Anionenrezeptor, das Porphyrin 2 wurde in $49 \%$ Gesamtausbeute durch Reaktion von Thymin mit dem entsprechenden Bromacetamidoporphyrin ${ }^{[10]}$ in DMSO mit $\mathrm{K}_{2} \mathrm{CO}_{3}$ als Base und anschließende Metallierung mit $\mathrm{Zn}(\mathrm{OAc})_{2} \cdot \mathrm{H}_{2} \mathrm{O}$ erhalten. Das $\mathrm{Zn}$-Metallzentrum in $\mathbf{2}$ sollte Anionen in aprotischen Lösungsmitteln binden können ${ }^{[11]}$. Wegen der Komplementarität der Diamidopyridin- und der Thymineinheit in 1 bzw. 2 sollte eine Assoziation über Wasserstoffbrückenbindungen möglich sein ${ }^{[12,13]}$.
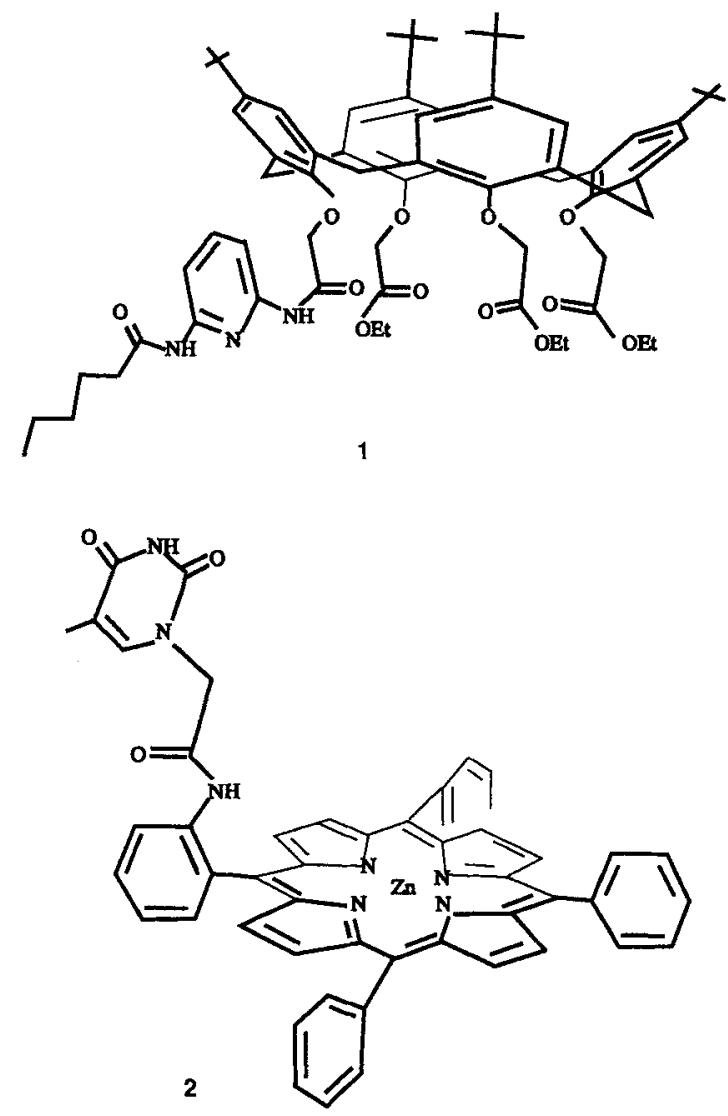

In $\mathrm{CDCl}_{3},\left[\mathrm{D}_{3}\right] \mathrm{MeCN}$ und $\left[\mathrm{D}_{8}\right]$ Toluol komplexiert das $\mathrm{Ca}$ lix[4]aren $1 \mathrm{Na}^{+}$-Ionen stark; durch Verdünnungsexperimente wurde die Assoziationskonstante $K_{\text {ass }}$ mit $\mathrm{NaNO}_{3}$ in $\left[\mathrm{D}_{3}\right] \mathrm{MeCN}$ zu $5.0 \times 10^{4} \mathrm{~mol}^{-1}$ bestimmt. Das Calix[4]aren 1 konnte einfach in die korrespondierenden lipophilen Komplexe $\left(\mathbf{1} \cdot \mathrm{Na}^{+}\right) \mathrm{ClO}_{4}^{-}$, $\left(1 \cdot \mathrm{Na}^{+}\right) \mathrm{I}^{-}$und $\left(1 \cdot \mathrm{Na}^{+}\right) \mathrm{SCN}^{-}$überführt werden, indem eine Lösung von 1 in $\mathrm{CH}_{2} \mathrm{Cl}_{2}$ mit einer gesättigten wäßrigen Lösung des entsprechenden Na-Salzes 3-5 h gerührt wurde. Mit den hydrophileren Salzen $\mathrm{NaF}$ und $\mathrm{NaH}_{2} \mathrm{PO}_{4}$ konnten keine Komplexe erhalten werden.

Überraschenderweise bildet das Diamidopyridinfragment in freiem 1 keine Wasserstoffbrückenbindungen zu komplementären Molekülen, wie $N$-Butylthymin. Die ${ }^{1} \mathrm{H}-\mathrm{NMR}$-Spektren zeigten bei Zugabe von $N$-Butylthymin zu Lösungen von 1 in $\mathrm{CDCl}_{3}$, $\left[\mathrm{D}_{3}\right] \mathrm{MeCN}$ oder $\left[\mathrm{D}_{8}\right]$ Toluol keine charakteristischen Verschiebungen $^{[12]}$ der $\mathrm{C}(\mathrm{O}) \mathrm{NH}$-Protonensignale. Offensichtlich werden in 1 zwischen der Diamidopyridingruppe und den Ethylcarboxylatgruppen intramolekulare Wasserstoffbrückenbindungen gebildet; die $\mathrm{C}(\mathrm{O}) \mathrm{NH}$-Protonensignale von 1 sind im Vergleich $\mathrm{zu}$ denen von $\left(\mathbf{1} \cdot \mathrm{Na}^{+}\right) \mathrm{ClO}_{4}^{-}$in $\mathrm{CDCl}_{3}$ oder 
$\left[\mathrm{D}_{3}\right] \mathrm{MeCN}$ um ca. $\Delta \delta=0.3$ bzw. 0.8 zu tieferem Feld verschoben. Die $\mathrm{Na}^{+}$-Komplexe von 1, in denen die drei Ester- und die porphyrinständige Amid-Carbonylgruppe das Kation koordinieren, bilden Wasserstoffbrückenbindungen zu komplementären Molekülen, was bei Zugabe von $N$-Butylthymin zu einer Lösung des Komplexes $\left(1 \cdot \mathrm{Na}^{+}\right) \mathrm{ClO}_{4}^{-}$in $\mathrm{CDCl}_{3},\left[\mathrm{D}_{3}\right] \mathrm{MeCN}$ oder $\left[\mathrm{D}_{8}\right]$ Toluol durch die Verschiebung der $\mathrm{C}(\mathrm{O}) \mathrm{NH}-\mathrm{Pro}-$ tonensignale des Diamidopyridins um $\Delta \delta=0.5-0.6$ zu tieferem Feld deutlich wird. Durch Verdünnungsexperimente in $\left[\mathrm{D}_{8}\right]$ Toluol wurden $K_{\text {ass }}$-Werte von $1.5 \times 10^{3} \mathrm{~mol}^{-1}(-\Delta G=$ $\left.17.8 \mathrm{~kJ} \mathrm{~mol}^{-1}\right)$ und $1.7 \times 10^{3} \mathrm{~mol}^{-1}\left(-\Delta G=18.1 \mathrm{~kJ} \mathrm{~mol}^{-1}\right)$ für $\left(1 \cdot \mathrm{Na}^{+}\right) \mathrm{ClO}_{4}^{-}$bzw. $\left(1 \cdot \mathrm{Na}^{+}\right) \mathrm{SCN}^{-}$bestimmt ${ }^{[14,15]}$. Die Fähigkeit von 1, Wasserstoffbrückenbindungen einzugehen, läßt sich also durch die Komplexierung von $\mathrm{Na}^{+}$-Ionen gleichsam ,einschalten“"[16]. Dies bedeutet, daß der Anionenrezeptor 2 grundsätzlich mit den Komplexen $\left(1 \cdot \mathrm{Na}^{+}\right) \mathrm{ClO}_{4}^{-}$oder $\left(1 \cdot \mathrm{Na}^{+}\right) \mathrm{SCN}^{-}$über die Diamidopyridin-Thymin-Wechselwirkung assoziiert werden kann. UV/Vis-Experimente mit dem Porphyrin 2 ergaben, daß dieses $\mathrm{I}^{-}$- und $\mathrm{SCN}^{-}$-Ion (eingesetzt als Tetrabutylammonium-Salze) in apolaren Lösungsmitteln $\left(\mathrm{CH}_{2} \mathrm{Cl}_{2}\right.$ und Toluol) komplexiert: Bei Zugabe von $\mathrm{Bu}_{4} \mathrm{~N}^{+} \mathrm{I}^{-}$ und $\mathrm{Bu}_{4} \mathrm{~N}^{+} \mathrm{SCN}^{-}$zu einer Lösung von 2 in Toluol wurde die Soret-Bande um 8-10 $\mathrm{nm}$ bathochrom verschoben. Dies ist im Einklang mit bekannten Daten für einfache Tetraphenylporphyrine ${ }^{111}$. Diese Assoziation ist nicht stark, und durch Verdünnungsexperimente wurde ein $K_{\text {ass }}$-Wert von ca. $10 \mathrm{~mol}^{-1}$ $\left(-\Delta G=5.6 \mathrm{~kJ} \mathrm{~mol}^{-1}\right)$ sowohl für das $\mathrm{I}^{-}-$als auch für das $\mathrm{SCN}^{-}$-Ion in Toluol erhalten. Für die Komplexierung von $\mathrm{ClO}_{4}^{-}$-Ionen gibt es keine Hinweise. Die Zugabe von freiem $1 \mathrm{zu}$ einer Lösung von 2 in Toluol führte zu keiner Veränderung der Anionenbindungseigenschaften von 2. Für die Komplexierung von $\mathrm{I}^{-}$- und $\mathrm{SCN}^{-}$-Ionen betragen die $K_{\text {ass }}$-Werte ca. $10 \mathrm{~mol}^{-1}$. Beim Mischen von 1 und 2 wurden ${ }^{1} \mathrm{H}$-NMR-spektroskopisch keine Wechselwirkungen zwischen ihren Wasserstoffbrückenbindungsstellen festgestellt.

Das entscheidende Assoziationsexperiment wurde mit $\left(1 \cdot \mathrm{Na}^{+}\right) \mathrm{SCN}^{-}$und 2 durchgeführt ${ }^{[17]}$. Die Zugabe von $2 \mathrm{zu}$ einer Lösung von $\left(1 \cdot \mathrm{Na}^{+}\right) \mathrm{SCN}^{-}$in $\left[\mathrm{D}_{8}\right]$ Toluol führte $\mathrm{zu}$ einer deutlichen Diamidopyridin-Thymin-Wechselwirkung: Aus den chemischen Verschiebungen der $\mathrm{C}(\mathrm{O})$-NH-Protonensignale wurde $K_{\text {ass }}=2.8 \times 10^{4} \mathrm{~mol}^{-1} \quad\left(-\Delta G=24.9 \mathrm{~kJ} \mathrm{~mol}^{-1}\right)$ für $\left(1 \cdot \mathrm{Na}^{+}\right)\left(2 \cdot \mathrm{SCN}^{-}\right)$berechnet. Dieser Wert ist deutlich größer als die für die separaten Wechselwirkungen in $\left(1 \cdot \mathrm{Na}^{+}\right) \cdot N$-Butylthymin $\cdot \mathrm{SCN}^{-}$und $\mathrm{Bu}_{4} \mathrm{~N}^{+}\left(2 \cdot \mathrm{SCN}^{-}\right)$. Durch eine Job-Auftragung wurde die 1:1-Stöchiometrie des Komplexes bestätigt $\left(\right.$ Abb. 1) ${ }^{[18.19]}$. Die Assoziation von $\left(1 \cdot \mathrm{Na}^{+}\right)\left(2 \cdot \mathrm{SCN}^{-}\right)$in Toluol wurde auch UV/Vis-spektroskopisch verfolgt: Die SoretBande der $\mathrm{Zn}-\mathrm{SCN}^{-}$-Wechselwirkung wird um $8 \mathrm{~nm}$ bathochrom verschoben (Abb. 2). Aus diesen Experimenten wurde ein $K_{\text {ass }}$-Wert von $2.5 \times 10^{4} \mathrm{~mol}^{-1}$ berechnet, der die starke Anionenbindung belegt.

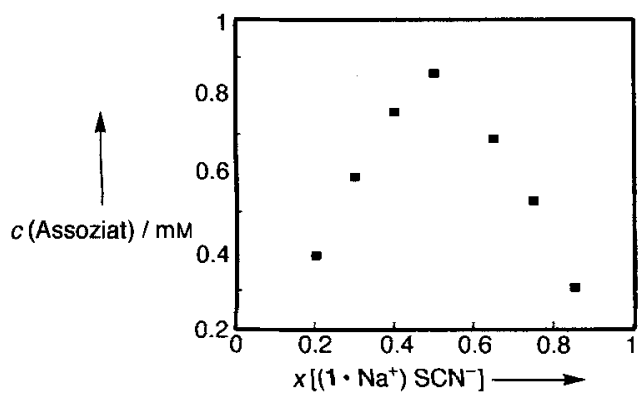

Abb. 1. Titration von $1 \mathrm{mmol}\left(\mathbf{1} \cdot \mathrm{Na}^{+}\right) \mathrm{SCN}^{-}$mit $1 \mathrm{mmol} 2$ in $\left[\mathrm{D}_{8}\right]$ Toluol unter Bildung des Assoziates $\left(1 \cdot \mathrm{Na}^{+}\right)\left(2 \cdot \mathrm{SCN}^{-}\right)$(Job-Auftragung).

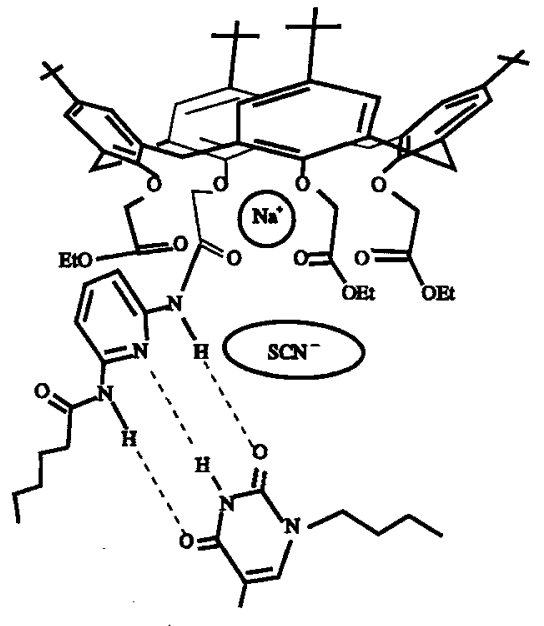

$\left(1 \cdot \mathrm{Na}^{+}\right) \cdot$ Thymin $\cdot \mathrm{SCN}^{-}$
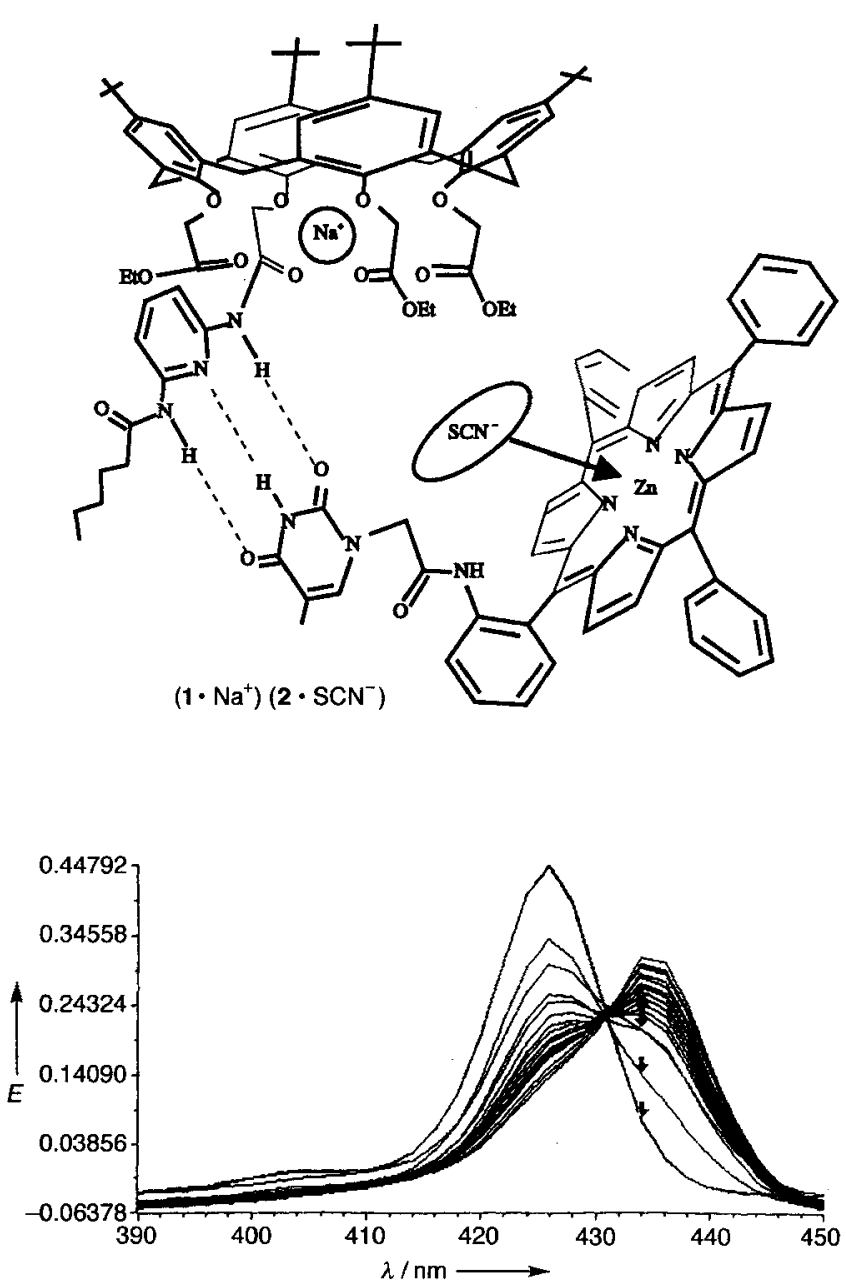

Abb. 2. Komplexierung von $\left(1 \cdot \mathrm{Na}^{+}\right) \mathrm{SCN}^{-}$mit 2 in Toluol. Die Assoziation wurde UV/Vis-spektroskopisch im Bereich zwischen 390 und 450 nm verfolgt (Extinktion $E$ ). [2] $=1 \times 10^{-3} \mathrm{~mm}$; die Konzentration von $\left(1 \cdot \mathrm{Na}^{+}\right) \mathrm{SCN}^{-}$wurde schrittweise von $5 \times 10^{-4}$ auf $1 \times 10^{-1} \mathrm{~mm}$ erhöht.

Diese Ergebnisse bestätigen die Bildung des nichtkovalent organisierten difunktionellen Rezeptors $\left(1 \cdot \mathrm{Na}^{+}\right)\left(2 \cdot \mathrm{SCN}^{-}\right)$ $(\mathrm{Abb} .3)^{[20]}$. Dabei wird das $\mathrm{Na}^{+}$-Ion durch das Calix[4]aren 1 und das $\mathrm{SCN}^{-}$-Ion durch das $\mathrm{Zn}$-Porphyrin 2 komplexiert. Der Kationen- und der Anionenrezeptor sind durch Aggregation über Wasserstoffbrückenbindungen miteinander verbunden. Die (deutliche) Verstärkung der Wasserstoffbrückenbindungen 


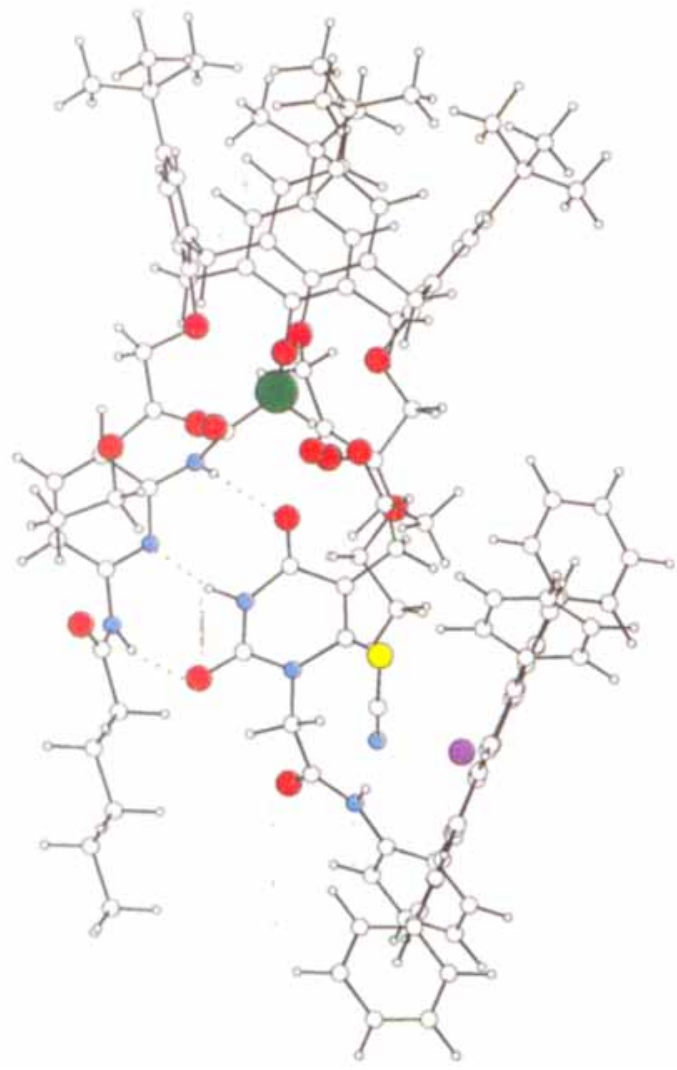

Abb. 3. Energieminimierte Struktur von $\left(1 \cdot \mathrm{Na}^{+}\right)\left(\mathrm{SCN}^{-} \cdot \mathbf{2}\right)$. Na: grün; $\mathrm{Zn}$ : violett; S: gelb; O: rot; $\mathrm{N}$ : blau; C, H: weiß.

sowie die Anionenkomplexierung deuten auf einen kooperativen Effekt der Wechselwirkungen zwischen dem Calix[4]aren 1, dem $\mathrm{Zn}$-Porphyrin 2 sowie dem $\mathrm{Na}^{+}$- und dem $\mathrm{SCN}^{-}$-Ion hin. Alle vier Komponenten des Komplexes sind in Lösung in ihren Positionen durch spezifische Ion-Dipol-Wechselwirkungen und Wasserstoffbrückenbindungen fixiert. Die Assoziation kann durch die Komplexierung des $\mathrm{Na}^{+}$-Ions durch den Rezeptor $\mathbf{l}$ „eingeschaltet" und einfach durch Zugabe von Methanol, das die Wasserstoffbrückenbindungen zerstört, „,ausgeschaltet" werden.

Eingegangen am 6. Mai $1995[$ Z 7965$]$

Stichworte: Molekulare Erkennung · Selbstorganisation · Wasserstoffbrücken

[1] a) J.-M. Lehn, Angew: Chem. 1990, 102, 1347-1362; Angew. Chem. Int. Ed Engl. 1990, 29, 1304-1319; b) G. M. Whitesides, J. P. Mathias, C. T. Seto, Science 1991. 254, 1312-1319; c) J. S. Lindsey, New J. Chem. 1991, 15, 153180.

[2] a) G. A. Ozin, Adv. Mater. 1992, 4, 612-649; b) S. L. Suib, Chem. Rev. 1993, 93, $803-826$

[3] a) M. C. Etter, Acc. (hem. Res. 1990, 23, 120-126; b) E. Fan, C. Vicent, S. J. Geib, A. D. Hamilton, Chem. Mater. 1994, 6, 1113-1117; c) J. C. MacDonald, G. M. Whitesides, Chem. Rey. 1994, 94, 2383-2420.

[4] a) J. L. Sessler, B. Wang. A. Harriman, J. Am. Chem. Soc. 1993, 115, 10418 10419 ; b) ibid. 1995, 117, 704-714.

[5] a) N. Branda, R. Wyler, J. Rebek, Jr., Science 1994, 263, 1267-1268; b) C. A Hunter, L. D. Sarson, Angew. Chem. 1994, 106, 2424-2426; Angew. Chem. Int Ed. Engl. 1994, 33, 2313-2316; c) N. Branda, R. M. Grotzfeld, C. Valdés, J. Rebek, Jr., J. Am. Chem. Soc. 1995, 117, 85-88.

[6] J. R. Granja, M. R. Ghadiri, J. Am. Chem. Soc. 1994, 116, 10785-10786, weitere Beispiele: a) O. F. Schall, K. Robinson, J. L. Atwood, G. W. Gokel, J Am. Chem. Soc 1993, 115, 5962-5969; b) O. F. Schall, G. W. Gokel, ibid. 1994, 116, 6089-6100.

[7] a) D. M. Rudkevich, Z. Brzozka, M. Palys, H. C. Visser, W. Verboom, D. N Reinhoudt, Angew. Chem. 1994, 106, 480-482; Angew. Chem. Int. Ed. Engl. 1994, 33, 467-468; b) D. M. Rudkevich, W. Verboom, D. N. Reinhoudt, $J$.
Org. Chem. 1994, 59,3683-3686; c) D. M. Rudkevich, J. D. Mercer-Chalmers, W. Verboom, R. Ungaro, F. de Jong, D. N. Reinhoudt, J. Am. Chem. Soc. 1995, 117, 6124-6125.

[8] F. Arnaud-Neu, E. M. Collins, M. Deasy, G. Ferguson, S. J. Harris, B. Kaitner, A. J. Lough, M. A. McKervey, E. Marques, B. L. Ruhl, M. J. SchwingWeill, E. Seward, J. Am. Chem. Soc. 1989, 111, 8681-8689.

[9] M. A. McKervey, M. Owens, H.-R. Schulten, W. Vogt, V. Böhmer, Angew. Chem. 1990, 102, 326-327; Angew. Chem. Int. Ed. Engl. 1990, 29, 280-- 282.

[10] S. Koeller, P. Cocolios, R. Guilard, New J. Chem 1994, 18, 849-859.

[11] M. Nappa, J. S. Valentine, J. Am. Chem. Soc. 1978, 100, 5075-5080.

[12] a) B. Feibusch, M. Sahha, K. Onan, B. Karger, R. Giese, J. Am. Chem. Soc. 1987, 109, 7531-7533; b) A. D. Hamilton, D. Van Engen, ibid. 1987, 109 $5035-5036$; c) A. V. Muehldorf, D. van Engen, J. C. Warner, A. D. Hamilton, ibid. 1988, 110, 6561-6562.

[13] Ausgewählte Daten von 1 : Schmp. $88-89^{\circ} \mathrm{C}(\mathrm{MeCN}) ;{ }^{1} \mathrm{H}-\mathrm{NMR}$ ([D, ]Toluol): $\delta=9.61,8.61(2$ br.s, $2 \mathrm{H}, \mathrm{NH}), 8.25,8.12\left(2 \mathrm{~d},{ }^{3} J(\mathrm{H}, \mathrm{H})=8.0 \mathrm{~Hz}, 2 \mathrm{H}\right.$, Diamidopyridin), $7.19\left(\mathrm{t},{ }^{3} J(\mathrm{H}, \mathrm{H})=8.0 \mathrm{~Hz}, 1 \mathrm{H}\right.$, Diamidopyridin), 6.92, 6.82, 6.69 $(3 \mathrm{~s}, 8 \mathrm{H}$, arom. $), 5.12,4.78\left(2 \mathrm{~d},{ }^{3} J(\mathrm{H}, \mathrm{H})=14.0 \mathrm{~Hz}, 4 \mathrm{H}, \mathrm{CH}, \mathrm{C}(\mathrm{O})\right), 4.98$, $4.72,3.17,3.10\left(4 \mathrm{~d},{ }^{3} J(\mathrm{H}, \mathrm{H})=13.0 \mathrm{~Hz}, 8 \mathrm{H}, \mathrm{CH}_{2}\right.$-Calix. $), 4.78,4.53(2 \mathrm{~s}, 4 \mathrm{H}$ $\left.\mathrm{CH}_{2} \mathrm{C}(\mathrm{O})\right), 4.9-4.5\left(\mathrm{~m}, 6 \mathrm{H}, \mathrm{OCH}_{2}\right), 2.45\left(\mathrm{t},{ }^{3} \mathrm{~J}(\mathrm{H}, \mathrm{H})=7.5 \mathrm{~Hz}, 2 \mathrm{H}\right.$, $\left.\mathrm{CH}_{2} \mathrm{C}(\mathrm{O}) \mathrm{N}\right), 1.85-1.75,1.4-1.3\left(2 \mathrm{~m}, 6 \mathrm{H}, \mathrm{CH}_{2}\right), 1.15(\mathrm{~s}, 18 \mathrm{H}, t \mathrm{Bu}), 1.09$ $1.03(2 \mathrm{~s}, 18 \mathrm{H}, t \mathrm{Bu}), 0.91\left(\mathrm{t},{ }^{3} J(\mathrm{H}, \mathrm{H})=7.2 \mathrm{~Hz}, 9 \mathrm{H}, \mathrm{CH}_{3}\right), 0.80\left(\mathrm{t},{ }^{3} J(\mathrm{H}, \mathrm{H})=\right.$ $\left.7.5 \mathrm{~Hz}, 3 \mathrm{H}, \mathrm{CH}_{3}\right)$; FAB-MS (NBA-Matrix): $m / z: 1154.4\left(M^{+}\right.$, ber. 1154.5$)$. Ausgewählte Daten von 2: Schmp. $253-255^{\circ} \mathrm{C}$; UV/Vis (Toluol): $\lambda_{\max }=426 \mathrm{~nm} ;{ }^{1} \mathrm{H}-\mathrm{NMR}\left(\left[\mathrm{D}_{8}\right]\right.$ Toluol $): \delta=9.06\left(\mathrm{~d},{ }^{3} J(\mathrm{H}, \mathrm{H})=8.0 \mathrm{~Hz}, 1 \mathrm{H}\right.$, arom.), $8.98\left(\mathrm{~s}, 4 \mathrm{H}\right.$, Porphyrin), 8.88, $8.67\left(2 \mathrm{~d},{ }^{3} \mathrm{~J}(\mathrm{H}, \mathrm{H})=4.8 \mathrm{~Hz}, 4 \mathrm{H}\right.$, Porphyrin), 8.3-8.0 (m, $8 \mathrm{H}$, arom. $), 7.80\left(\mathrm{~d},{ }^{3} J(\mathrm{H}, \mathrm{H})=8.0 \mathrm{~Hz}, 1 \mathrm{H}\right.$, arom. $7.6-7.4(\mathrm{~m}, 10 \mathrm{H}$, arom. $+\mathrm{NH}), 7.09\left(\mathrm{t},{ }^{3} J(\mathrm{H}, \mathrm{H})=7.2 \mathrm{~Hz}, 1 \mathrm{H}\right.$, arom. $), 5.30$ (s, $1 \mathrm{H}$, Thymin), $2.70\left(\mathrm{~s}, 2 \mathrm{H}, \mathrm{NCH}_{2} \mathrm{C}(\mathrm{O})\right), 2.01\left(\mathrm{~s}, 3 \mathrm{H}, \mathrm{CH}_{3}\right)$; FAB-MS (NBAMatrix): $m / z: 859.1\left(M^{+}\right.$, ber. 859.3$)$ - - NBA = 3-Nitrobenzylalkoho].

[14] Alle $K_{\text {ass }}$-Werte wurden bei $293 \mathrm{~K}$ erhalten. Die Konzentration an $\left(\mathbf{1} \cdot \mathrm{Na}^{+}\right)$ betrug konstant $1.00 \mathrm{mmol}$, die Konzentration an Gast variierte im Bereich von $0.25-5.00 \mathrm{mmol}$. Die Werte wurden durch nichtlineare Regression analog dem von J. A. A. de Boer, D. N. Reinhoudt, S. Harkema, G. J. van Hummel, F. de Jong, J. Am. Chem. Soc. 1982, 104, 4073-4076, beschriebenen Verfahren berechnet (Fehler $\pm 5 \%)$.

[15] Der Komplex $\left(1 \cdot \mathrm{Na}^{+}\right) \mathrm{I}^{-}$ist sehr lichtempfindlich und zersetzte sich während der Titration.

[16] Über ein ähnliches Phänomen wurde berichtet: H. Murakami, S. Shinkai, Chem. Soc. Chem. Commun. 1993, 1533-1535.

[17] Das Porphyrin 2 komplexiert $\mathrm{F}^{-}$-Ionen (eingesetzt als TetrabutylammoniumSalz) sehr stark; für $K_{\text {ass }}$ wurde in Toluol ein Wert von $2.3 \times 10^{4} \mathrm{~mol}^{-1}$ berechnet. Da der Komplex $\left(1 \cdot \mathrm{Na}^{+}\right) \mathrm{F}^{-}$nicht erhalten werden konnte, wurde eine Lösung von $\left(\mathbf{1} \cdot \mathrm{Na}^{+}\right)\left(\mathbf{2} \cdot \mathrm{ClO}_{4}^{-}\right)$in Toluol mit $\mathrm{BuN}_{4}^{+} \mathrm{F}^{-}$titriert. Zwar wurde dabei anfänglich eine starke Wechselwirkung $\left(K_{\mathrm{ass}} \approx 3.0 \times 10^{4} \mathrm{~mol}^{-1}\right)$ detektiert (UV/Vis, ${ }^{1} \mathrm{H}-\mathrm{NMR}$ ), doch fiel dann rasch NaF aus, wodurch eine weitere Assoziation verhindert wurde.

[18] K. A. Connors, Binding Constants, Wiley, New York, 1987, S. 24.

[19] ${ }^{1} \mathrm{H}-\mathrm{NMR}-\mathrm{Spektrum}$ von $\left(\mathbf{1} \cdot \mathrm{Na}^{+}\right)\left(2 \cdot \mathrm{SCN}^{-}\right)\left(\left[\mathrm{D}_{8}\right]\right.$ Toluol $): \delta=10.29,10.18$ ( 2 br.s, $2 \mathrm{H}, \mathrm{NH}), 8.99$ (s, $4 \mathrm{H}$, Porphyrin), $8.95,8.26\left(2 \mathrm{~d},{ }^{3} \mathrm{~J}(\mathrm{H}, \mathrm{H})=8.0 \mathrm{~Hz}\right.$, $2 \mathrm{H}$, arom. $), 8.83\left(\mathrm{q},{ }^{3} J(\mathrm{H}, \mathrm{H})=4.8 \mathrm{~Hz}, 4 \mathrm{H}\right.$, Porphyrin $), 8.50\left(\mathrm{~d},{ }^{3} J(\mathrm{H}, \mathrm{H})=\right.$ $8.0 \mathrm{~Hz}, 2 \mathrm{H}$, arom. $), 8.2-8.0,7.8-7.5(2 \mathrm{~m}, 18 \mathrm{H}$, arom. $+\mathrm{NH}), 7.38(\mathrm{t}$ ${ }^{3} J(\mathrm{H}, \mathrm{H})=8.0 \mathrm{~Hz}, 1 \mathrm{H}$, arom. $), 7.25,7.17(2 \mathrm{~s}, 8 \mathrm{H}$, arom. $), 5.60(\mathrm{~s}, 1 \mathrm{H}$, Thy $\min ), \quad 4.60, \quad 4.22\left(2 \mathrm{~s}, \quad 4 \mathrm{H}, \quad \mathrm{CH}_{2} \mathrm{C}(\mathrm{O})\right), 4.55,4.49, \quad 3.30, \quad 3.25 \quad(4 \mathrm{~d}$, ${ }^{3} J(\mathrm{H}, \mathrm{H})=13.0 \mathrm{~Hz}, 8 \mathrm{H}, \mathrm{CH}_{2}$-Calix. $), 4.38,4.11\left(2 \mathrm{~d},{ }^{3} J(\mathrm{H}, \mathrm{H})=14.0 \mathrm{~Hz}, 4 \mathrm{H}\right.$, $\left.\mathrm{CH}_{2} \mathrm{C}(\mathrm{O})\right), 4.0-3.8\left(\mathrm{~m}, 6 \mathrm{H}, \mathrm{OCH}_{2}\right), 3.28\left(\mathrm{~s}, 2 \mathrm{H}, \mathrm{NCH}_{2} \mathrm{C}(\mathrm{O})\right), 2.53(\mathrm{t}$ $\left.{ }^{3} J(\mathrm{H}, \mathrm{H})=7.5 \mathrm{~Hz}, 2 \mathrm{H}, \mathrm{CH}_{2} \mathrm{C}(\mathrm{O}) \mathrm{N}\right), 2.01\left(\mathrm{~s}_{1} 3 \mathrm{H}, \mathrm{CH}_{3}\right), 1.85-1.75,1.4-1.1$ $\left(2 \mathrm{~m}, 6 \mathrm{H}, \mathrm{CH}_{2}\right), 1.21(\mathrm{~s}, 36 \mathrm{H}, t \mathrm{Bu}), 1.03,1.00(2 \mathrm{t}, 3 \mathrm{~J}(\mathrm{H}, \mathrm{H})=7.2 \mathrm{~Hz}$, $\left.{ }^{3} J(\mathrm{H}, \mathrm{H})=7.5 \mathrm{~Hz}, 6 \mathrm{H}, \mathrm{CH}_{3}\right), 0.80\left(\mathrm{t},{ }^{3} J(\mathrm{H}, \mathrm{H})=7.2 \mathrm{~Hz}, 6 \mathrm{H}, \mathrm{CH}_{3}\right)$

[20] Die vorgeschlagenen Strukturen wurden durch Molecular-Mechanics-Rechnungen (Quanta/CHARMm Version 3.3) bestätigt. Energieminimierungen (conjugate gradient) wurden durchgeführt (Steepest Descents gefolgt von Adopted Based Newton Raphson), bis der quadratische Mittelwert des Gradienten kleiner als $0.01 \mathrm{kcal} \mathrm{mol}^{-1} \AA^{-1}$ war. 